\title{
Reconstrucción alveolar de maxilar atrófico con injerto de calota craneal. A propósito de dos casos
}

\section{Alveolar bone reconstruction of atrophic maxilla with calvarial bone graft. Two case reports}

\author{
MODELO PÉREZ A* \\ RENDÓN INFANTE I** \\ BUDIÑO CARBONERO SM**
}

Modelo Pérez A, Rendón Infante I, Budiño Carbonero SM. Reconstrucción alveolar de maxilar atrófico con injerto de calota craneal.A propósito de dos casos. Av Periodon Implantol. 2009; 21, 3: 141-144.

\begin{abstract}
RESUIMEN
La atrofia alveolar del maxilar superior es un reto difícil de solventar en la práctica clínica para la rehabilitación dental sobre implantes. Existen varios métodos de reconstrucción con dicha finalidad. Aunque la elección del método depende de varios factores, de los más importantes son la cantidad de hueso remanente y los deseos del paciente. Presentamos dos casos en los que hemos efectuado la rehabilitación prostodóncica sobre implantes MG Osseous, no removible en uno y removible en el otro. Previa a la colocación de los implantes se llevó a cabo la reconstrucción alveolar con injerto óseo de calota craneal.
\end{abstract}

PALABRAS CLAVE: Alveolar, injerto óseo, atrofia maxilar, calota craneal, implantes.

\section{SUMMARY}

The alveolar atrophy of superior maxilla is a difficult challenge to resolve in clinical practice for the dental rehabilitation on implants. There are several methods of reconstruction with this purpose. Although the election of the method depends on several factors, most importantly the amount of bone surplus and the desires patient's. We presented/displayed two cases in which we have carried out the prosthodontist rehabilitation on implants MG Osseous, nonremovable in one and removable in the other. Previous to the positioning of the implants alveolar reconstruction was carried out with calvarial bone graft.

KEY WORDS: Alveolar, bone graft, maxillary atrophic, calvarial bone, implants.

Fecha de recepción: 18 de septiembre de 2008.

Fecha de aceptación: 20 de octubre de 2008.

\section{INTRODUCCIÓN}

La atrofia alveolar del maxilar superior es un hallazgo habitual en las personas que han sufrido pérdidas dentarias durante varios años por distintas causas como postexodoncia, enfermedad periodontal, traumatismos o cirugía ablativa. Un gran número de autores estiman que el mínimo de altura ósea para la colocación de un implante sea de $10 \mathrm{~mm}$ y de $1 \mathrm{~mm}$ a cada lado respecto a la anchura. Estos hechos apuntan que en muchos de estos casos son necesarias técnicas de reconstrucción alveolar para intentar una restauración prostodóncica lo más estética y funcional posible.

Actualmente la técnica de reconstrucción alveolar con injertos óseos es una de las más usadas, siendo los in-

\footnotetext{
* Doctor en Medicina y Cirugía General. Médico Especialista en Cirugía Oral y Maxilofacial. Hospital Universitario Puerta del Mar. Cádiz. España. Unidad de Cirugía Oral y Maxilofacial.

** Médico Especialista en Cirugía Oral y Maxilofacial. Hospital Puerta del Mar. Cádiz. España.
} 
jertos óseos autógenos los que proporcionan mejores resultados.

Existen distintos tipos de autoinjertos óseos con características propias de cada uno de ellos. Presentamos dos casos de reconstrucción alveolar maxilar superior con calota craneal.

\section{CASO CLÍNICO 1}

La paciente GGP de 43 años, presentaba una atrofia severa de maxilar (Fig. 1B) superior que fue constatada de forma clínica y mediante Dentascan. La paciente expresó su deseo de portar una prótesis dentaria no removible. Tras su consentimiento informado, bajo anestesia general se practicó la toma de autoinjerto de calota craneal en bloques, desdoblando la tabla externa de la región parietal en cantidad abundante (Fig. 1A). Los bloques se toman con fresas y sierras oscilantes, recíprocamente y circulares. Es aprovechada toda la viruta ósea de su extracción para empaquetarla junto al bloque en el lecho receptor. Su posterior colocación en onlay en la creta alveolar y en inlay bajo los senos maxilares (1), fijados (2) con tornillos de osteosíntesis de titanio. Para ello se efectuó un gran colgajo desde 4-5 mm de palatino, despegamiento amplio mucoperióstico hasta 3-4 $\mathrm{mm}$ por encima de la mitad de los senos maxilares para evitar tensión en la sutura.

Aunque hay autores que colocan los implantes en el mismo acto quirúrgico $(3,4)$, en este caso se esperó tres meses, colocando un total de diecinueve implantes MG OSSEOUS de hexágono externo. Nueve de 3,4 $\mathrm{mm}$ de diámetro distribuidos en maxilar superior (Figs. 1C y D) y diez, de 3,3, 3,4 y 3,75 mm de diámetro en mandíbula. Tras dejar tres meses y medio como periodo de integración, se inició la fase prostodóncica (Fig. 1E). Los resultados funcionales y estéticos fueron considerados como buenos por la paciente (Figs. IF, G y H). La paciente se encuentra con 18 meses de periodo de seguimiento con estabilidad en el tratamiento efectuado.

\section{CASO CLÍNICO 2}

La paciente IGG de 45 años, presentaba atrofia maxilar superior de características similares al caso 1 , constatada clínicamente y Dentascan. Se objetivaba una depresión subnasal muy marcada, y se ofertó como una de las posibilidades de tratamiento una prótesis sobre implantes removible, para proyectar hacia adelante el labio superior. La paciente había rechazado tratamiento con Le Fort I.

Tras consentimiento informado, igual que en el caso 1 , se practicó una incisión hemicoronal parcial para la toma de injerto óseo de calota craneal en bloque de la zona parietal (Fig. 2A) y su colocación en onlay por palatino (Fig. 2B) de 16 a 26 fijados con tornillos de titanio. En este caso la incisión fue 2-3 $\mathrm{mm}$ por debajo del fondo del vestíbulo superior, con despegamiento amplio mucoperióstico.
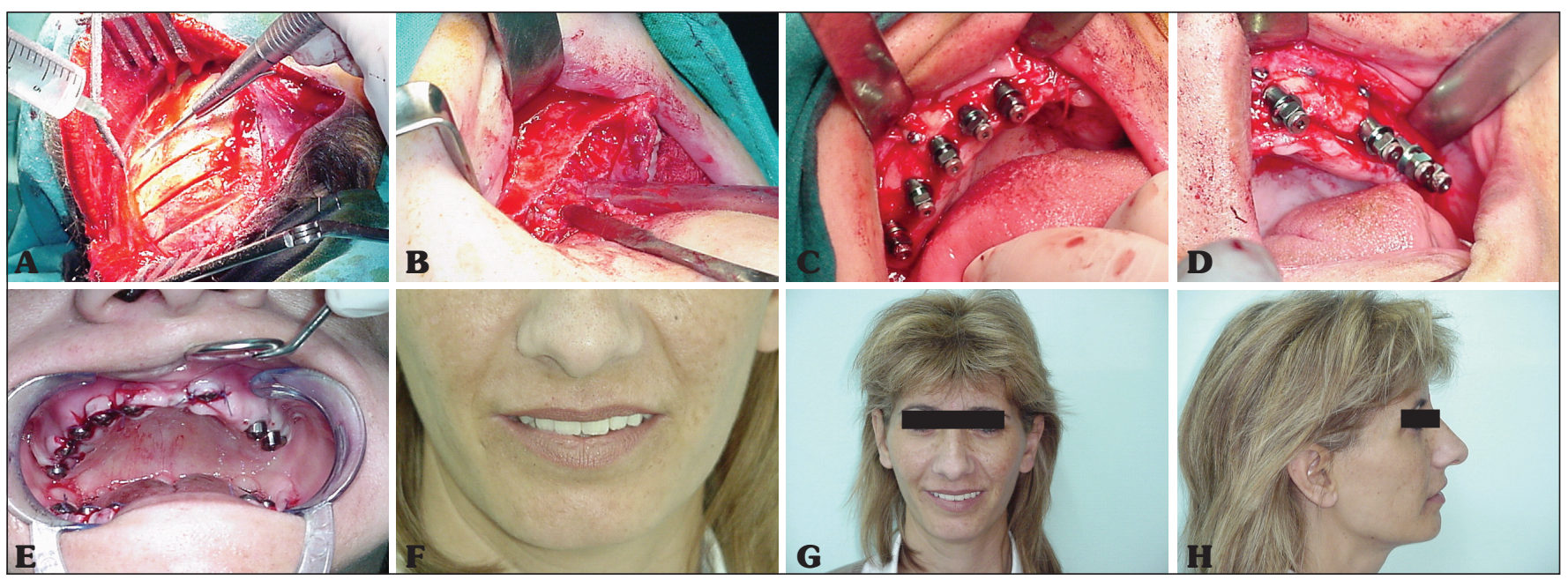

Fig. 1: A. Se observa la toma de injerto de calota parietal. B. El hueso alveolar atrófico muy fino. C y D. Colocación de los implantes MG Osseous en maxilar derecho e izquierdo. E. Pilares de cicatrización. $\boldsymbol{F}, \boldsymbol{G}$ y $\boldsymbol{H}$. Resultados en la paciente. 


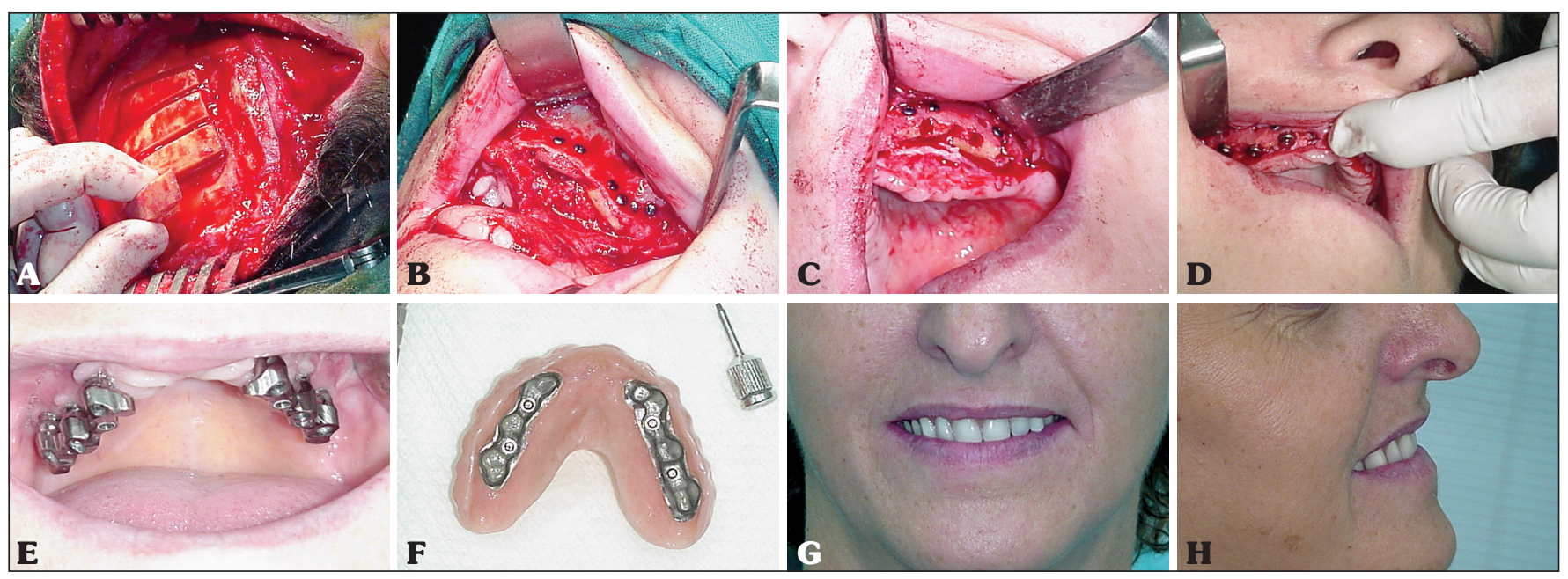

Fig. 2: $\boldsymbol{A}$. Se muestra desde la toma de bloque de calota craneal. B. Osteosíntesis con tornillos de titanio. C. Reentrada. D. Colocación de los implantes. E. Estructura. F. Prótesis dental. G y H. Resultado en la paciente.

Tras esperar tres meses, se hizo la reentrada (Fig. 2C) para colocar los seis implantes MG OSSEOUS de $3,3 \mathrm{~mm}$ de diámetro, tres en el primer cuadrante y otros tres en el segundo cuadrante (Figs. 2D y E). Pasado el periodo de integración de tres meses y medio, se llevó a cabo la fase prostodóncica cuyos resultados se muestran en las figuras $2 F-H$. Tras 20 meses de seguimiento la paciente está satisfecha con el tratamiento y estable en cuanto a la osteointegración de los implantes.

\section{DISCUSIÓN}

En la revisión publicada por Colman (5) la supervivencia de los implantes en maxilar superior oscila entre 83-91\% dependiendo de la colocación del injerto óseo en onlay, inlay u onlay junto a inlay. Los casos presentados de atrofia maxilar podían haberse tratado por otros procedimientos (4-12). Estos podrían incluir desde otra zona donante del autoinjerto, cambio en la base ósea con Le Fort I (4), reconstrucción con mallas de titanio, osteotomía expansiva alveolar (13) y la aplicación de distracción ósea. La osteotomía expansiva y la distracción alveolar se descartaron por el escaso grosor de reborde alveolar que existía. La técnica de Le Fort I fue rechazada por las pacientes. Respecto a la zona donante existen otras como cresta ilíaca, sínfisis mentoniana, rama ascendente mandibular, tuberosidad maxilar y tibia. De estas, dado que necesitábamos gran cantidad de hueso, descartamos las que proporcionaban escasa cantidad y decidimos la calota craneal porque aportaba ventajas (14). Entre las ventajas de la calota craneal como zona donante, destacamos la ausencia de dolores postoperatorios (en comparación con la cresta ilíaca), cicatriz poco o nada visible, menor reabsorción $(15,2)$ del hueso membranoso (calota) en comparación con el endocondral (cresta ilíaca). El autoinjerto de calota craneal es considerado de primera elección por muchos autores para las reconstrucciones con implantes. La principal desventaja es la escasa moldeabilidad debido a su gran componente cortical. Aunque están descritas complicaciones como hematoma epidural o subdural, fístula de líquido cefalorraquídeo y daño cerebral, éstas son muy poco frecuentes con el uso de fresas y sierras oscilantes, reciprocantes y circulares.

\section{CONCLUSIONES}

La atrofia severa del maxilar superior puede tratarse por distintos métodos para la rehabilitación prostodóncica con implantes. En los casos en los que se precisa aportar gran cantidad de injerto óseo consideramos que la calota craneal es el de primera elección por sus ventajas, de las que cabe destacar la escasa reabsorción y estabilidad del implante. Esto proporciona una tasa de supervivencia superior al $80 \%$ de los implantes.

\section{BIBLIOGRAFÍA}

1. Small SA, Sinner ID, Pane FV, Shapiro HJ, Stein JI. Augmenting the maxillary sinus for implants: Report 
of 27 patients. Int J Oral Maxillofac Implants. 1993;8: 523-8.

2. Phililps JH, Rahn BA. Fixation effects on membranous and endocondral onlay bone-graft resorption. Plast Reconstr Surg 1990;85:891.

3. Isakson S, Alberius P. Maxillary alveolar ridge augmentation with onlay bone-grafts and immediate endosseous implants. J Craniomaxillofac Surg 1992;20:2-7.

4. Li KK, Stephens Wl, Gliklich R. Reconstruction of severely atrophic edentulous maxilla using Le Fort I osteotomy with simultaneous bone graft and implant placement. J Oral Maxillofac Surg 1996;54:542-6.

5. Tolman DE. Reconstructive procedures with endosseous implants in grafted bone: A review of the literature. Int J Oral Maxillofac Implants 1995; 10:275-94.

6. Jensen J, Sindet-Pedersen S, Oliver AJ.Varying treatment strategies for reconstruction of maxillary atrophy with implants: Results in 98 patients. J Oral Maxillofac Surg 1994;52:210-6.

7. Lustmann J, Lewinstein I. Interpositional bone grafting technique to widen narrow maxillary ridge. Int J Oral Maxillofac Implants 1995;10:568-77.

8. Adell R, Eriksson B, Lekholm U, Branemark PL, Jemt T. Longterm follow-up study of osseointegrated implants in the treatment of totally edentulous jaws. Int J Oral Maxillofac Implants 1190;5:347-59.

9. Adell R, Lekholm U, Grondahl K, Branemark Pl, Lindstrom J, Jacosson M. Reconstruction of severely resorbed edentulous maxillae using osseointegrated fixtures in immediate autogenous bone grafts. Int J Oral Maxillofac Implants 1990;5:233-46.
10 Ahlqvist J, Borg K, Gunne J, Nilson H, Olsson M, Astrand P. Osseointegrated implants in edentulous jaws: 2-year longitudinal study. Int J Oral Maxillofac Implants 1990;5:155-63.

11. Breine U, Branemark Pl. Reconstruction of alveolar jaw bone: an experimental and clinical study of immediate and preformed autologous bone grafts in combination with osseointegrated implants. Scan J Plas Reconstr Surg 1980;14 23-8.

12. Collins TA, Brown GK, Johson N, Massey JA, Nunn BD. Team management of atrophic edentulism with autogenous inlay, veneer, and split grafts and endosseous implants: Case reports. Quintessence Int 1995;26:79-93.

13. Summers. The osteotome technique: Part 2 . The ridge expansion osteotomy (REO) procedure. Compend Contin Educ dent 1995;15:422-36.

14. Donovan MG, Dickerson NC, Hanson LJ, Gustafson RB. Maxillary and mandibular reconstruction using calvarial bone grafts and Branemark implants: A preliminary report. J Oral Maxillofac Surg 1994;52:588-94.

15. Dado DV, Izquierdo R. Absorption of onlay bone grafts in immature rabbits: membranous versus endocondral bone and bone struts versus paste. Ann Plast Surg 1989;23:39.

\section{CORRESPONDENCIA}

Antonio Modelo Pérez

Pérgolas, $3,1^{\circ} \mathrm{C}$

11007 Cádiz

e-mail: amodelop@hotmail.com 